\title{
Miotoxicidade por organofosforados
}

\section{Organophosphate myotoxicity}

\author{
Maria J. Cavaliere, Edenilson E. Calore, Nilda M. Perez e Flávio Rodrigues Puga \\ Divisão de Patologia do Insituto Adolfo Lutz. São Paulo, SP - Brasil (M.J.C.); Seção de Anatomia \\ Patológica do Instituto Emílio Ribas. São Paulo, SP - Brasil (E.E.C., N.M.P.); Seção deToxicologia e \\ Higiene Comparada do Instituto Bilógico. São Paulo, SP - Brasil (F.R.P.)
}

\begin{abstract}
Resumo
Os organofosforados são um grupo de compostos químicos amplamente utilizados em agropecuária como inseticidas, ocasionando intoxicações acidentais em animais e humanos, e mesmo sendo utilizados em tentativas de suicídio. A toxicidade desses produtos decorre sobretudo de insuficiência cárdio-respiratória por compromentimento do sistema nervoso autônomo. Sabe-se que alguns destes compostos induzem em animais de experimentação e em humanos, uma miopatia caracterizada por degeneração de células musculares, comprometendo sobretudo a musculatura respiratória. Baseado no fato de que este comprometimento contribui para a piora da função respiratória, propõe-se um protocolo de avaliação rotineira de miotoxicidade por compostos organofosforados, através de uma bateria mínima e suficiente de colorações e reações histoquímicas para quantificação da necrose muscular. Utilizaram-se como modelo experimental, grupos de ratos albinos (Wistar) intoxicados com o organofosforado paraoxon, com e sem antídotos (atropina ou pralidoxima). Verificou-se nos grupos tratados com paraoxon e paraoxon mais atropina, necrose de fibras musculares no diafragma, que atingia em determinadas áreas até $15 \%$ das fibras. No grupo tratado com paraoxon mais pralidoxima, a necrose foi mínima, evidenciando o papel mioprotetor deste último antídoto.
\end{abstract}

Inseticidas organofosforados, toxicidade. Fibras musculares, patologia.

\begin{abstract}
s
Organophosphates comprise a group of chemical compounds extensively used in farming as insecticides, which cause accidental poisoning in animals and men and are also used in suicide attempts. The toxicity of these compounds is due especially to the cardiac and respiratory impairment in consequence of autonomic nervous system disorders. However, it is known that some of these products induce a myopathy in experimental animals and humans. This myopathy is characterized by muscle cell degeneration, involving above all the respiratory muscles. Based on the fact that this involvement certainly enhances the respiratory impairment, this study offers an experimental method for routine evaluation of organophosphate myotoxicity, using a minimal and sufficient battery of stains and histochemical reactions, for muscle necrosis quantification. For this purpose, albino rats (Wistar) treated with the
\end{abstract}

Correspondência para / Correspondence to: Maria José Cavaliere - Divisão do Patologia do Instituto Adolfo Lutz. Av. Dr. Arnaldo, 355 - $01246-902$ São Paulo, SP - Brasil.

Edição subvencionada pela FAPESP. Processo 95/2290-6.

Recebido em 30.6.1995. Aprovado em 8.2.1996. 
organophosphate paraoxon, were used both with and without antidotes (atropine or pralidoxime). Muscle fiber necrosis in the diaphragm of the rats treated with paraoxon or paraoxon and atropine, that affected about $15 \%$ of the fibers in some areas, was detected. In the group treated with paraoxon and pralidoxime, a minimal necrosis was seen, revealing a protective role of this later antidote during the development of myopathy.

Insecticides, organophosphate, toxicology. Muscle fibers, pathology.

\section{INTRODUÇÃO}

Os pesticidas organofosforados atuam inibindo as colinesterases, principalmente a acetilcolinesterase (AchE; EC3.1.1.7), aumentando o nível de acetilcolina nas sinapses. Em mamíferos, estes efeitos caracterizam-se principalmente por lacrimejamento, salivação, sudorese, diarréia, tremores e distúrbios cardiorrespiratórios. Estes últimos são decorrentes de broncoconstricção, aumento das secreções brônquicas e bradicardia, bem como de depressão do sistema nervoso central, sendo as principais causas de morbidade e mortalidade por tais produtos ${ }^{11}$.

Alterações estruturais e funcionais observadas nos músculos esqueléticos estão relacionadas com as estruturas químicas dos produtos e com o tipo de músculo, sendo o diafragma o mais afetado em estudos experimentais $\mathrm{s}^{3,4,5,8,9,13,14,15}$. Necrose muscular, semelhante à observada em animais de laboratório, foi encontrada em músculos diafragma e intercostais de indivíduos intoxicados pelo parathion ${ }^{6}$, diazinon $^{1}$, triclornato ${ }^{7}$ e combinação de malathion e diazinon ${ }^{18}$. Uma vez que a insuficiência respiratória é uma das consequiências mais graves das intoxicações por organofosforados, é possível que o envolvimento dos músculos da respiração constitua agravante do distúrbio respiratório, o qual é devido à disfunção do sistema nervoso autônomo.

O tratamento das intoxicações por organofosforados baseia-se principalmente no uso da atropina, antídoto sintomático e, com menor freqüência, das oximas, antídotos específicos que reativam as colinesterases.

No Brasil, a ocorrência de intoxicações por pesticidas do grupo dos organofosforados continua sendo alta, apesar de seu uso ter diminuído em relação à década dos anos oitenta. Os dados estatísticos dos Centros de Toxicologia de Belo Horizonte, Campinas, Florianópolis, Ribeirão Preto, Londrina e Maringá mostram que de 495 casos de intoxicações ocupacionais, cerca de $34,9 \%$ foram devidos a organofosforados; de 622 casos de tentativas de suicídio, $38,1 \%$ resultaram do uso de compostos deste grupo; de 38 casos de óbitos, $44,7 \%$ foram devidos a organofosforados ${ }^{16}$.
Os objetivos do presente trabalho foram:

1 - padronizar técnicas histoquímicas para avaliar lesões musculares causadas por organofosforados;

2 - comparar a eficácia dos antídotos atropina e pralidoxima na prevenção de tais lesões.

\section{MATERIAL E MÉTODO}

\begin{abstract}
Animais
Ratos albinos machos e fêmeas da linhagem Wistar $(225 \mathrm{~g} \pm 25 \mathrm{~g}$ ) foram divididos em 4 grupos. Cada grupo foi constituído por 8 animais (4 machos e 4 fêmeas), mantidos separados durante todo o experimento em gaiolas de plástico, recebendo água e ração ad libitum.
\end{abstract}

\section{Produtos}

O organofosforado utilizado foi o Paraoxon ${ }^{\circledR}$ (dietilparanitrofenil fosfato) com 98,2\% de pureza, fornecido pela Bayer do Brasil, São Paulo. O Sulfato de Atropina ${ }^{\circledR}$ foi proveniente da Sigma e o mesilato de pralidoxima (Contrathion) ${ }^{\circledR}$, da Rhodia. O paraoxon foi dissolvido em propilenoglicol $(0,3 \mathrm{mg} / \mathrm{ml})$; a atropina $(0,3 \mathrm{mg} / \mathrm{ml})$ e a pralidoxima $(10,0 \mathrm{mg} / \mathrm{ml})$ foram preparadas em solução fisiológica.

\section{Administração de Paraoxon, Atropina e Pralidoxima}

Os produtos foram injetados via intraperitoneal por 4 dias com intervalos de $24 \mathrm{~h}$. O grupo 1 foi tratado apenas com paraoxon $(0,3 \mathrm{mg} / \mathrm{kg})$; o grupo $2 \mathrm{com}$ paraoxon $(0,3$ $\mathrm{mg} / \mathrm{kg})$ seguido imediatamente de atropina $(0,3 \mathrm{mg} / \mathrm{kg}) ; \mathrm{o}$ grupo $3 \mathrm{com}$ paraoxon $(0,3 \mathrm{mg} / \mathrm{kg})$ seguido imediatamente de pralidoxima $(10,0 \mathrm{mg} / \mathrm{kg})$ e o grupo 4 (Controle) recebeu apenas propilenoglicol $(0,25 \mathrm{ml})$.

\section{Observação dos Sinais de Intoxicação}

Os sinais de intoxicação e sua intensidade foram observados diariamente após a aplicação dos produtos e registrados durante a primeira hora e a cada $30 \mathrm{~min}$ das seis horas subsequientes. Para o registro da intensidade dos sinais de intoxicação estabeleceu-se o seguinte critério: (+) leve, $(++)$ moderado e $(+++)$ intenso.

\section{Determinação da Atividade da Colinesterase Plasmática}

Amostras de $1 \mathrm{ml}$ de sangue foram colhidas por punção do plexo retro-orbitário, 24 h após o último trata- 
mento. O plasma foi separado por centrifugação a 2000 r.p.m. por $15 \mathrm{~min}$, e a atividade da colinesterase foi determinada pelo método espectrofotométrico de Ellman ${ }^{12}$, 1961 modificado por Wilheim ${ }^{19}, 1967$. O substrato utilizado foi a acetiltiocolina e a atividade enzimática foi determinada pela variação de absorbância/ml/minuto $(\Delta \mathrm{E} / \mathrm{ml} / \mathrm{min})$, sendo feitas leituras no comprimento de onda de $430 \mathrm{~nm}$.

\section{Técnicas Histológicas e Histoquímicas}

Os animas foram anestesiados com éter e decapitados $24 \mathrm{~h}$ após o último tratamento e os hemidiafragmas esquerdos foram colhidos e congelados em n-hexano previamente resfriado em nitrogênio líquido, em posição para obter cortes transversais em micrótomo-criostato. Cortes seriados ( $8 \mu \mathrm{m}$ de espessura), colhidos em diferentes lâminas para microscopia, foram corados pela hematoxilina-eosina (HE) bem como submetidos às reações histoquímicas para fosfatase ácida e nicotinamida-adeninadinucleotídio-tetrazolium-redutase (NADH-TR) segundo Dubowitz ${ }^{10}, 1985$.

\section{Avaliação das Alterações Histológicas}

Foi observada ao microscópio óptico toda a área do corte histológico e feita semiquantificação das alterações encontradas, seguindo os seguintes critérios: 1) observação pelo HE, no aumento de $100 \mathrm{X}$, com delimitação dos 5 campos mais alterados; 2) semi-quantificação de fibras alteradas nos 5 campos, no aumento de $400 \mathrm{X}$, nos cortes seriados submetidos às reações histoquímicas de NADHTR e fosfatase ácida, sendo (+) até 5\% de fibras alteradas; $(++)$ de 5 a $15 \%$ de fibras alteradas e (+++) acima de $15 \%$ de fibras alteradas.

\section{Análise Estatística}

Os resultados da atividade da colinesterase plasmática foram analisados pelo teste de Mann-Whitney-Wilcoxon para amostras não-paramétricas, tomando-se como índice de significância $\mathrm{p}<0,01$.

\section{RESULTADOS}

\section{Sinais de Intoxicação}

A Tabela 1 mostra os sinais de intoxicação observados. O grupo que recebeu apenas paraoxon (grupo 1) apresentou os sinais mais intensos. A aplicação de atropina diminuiu a intensidade dos sinais, com exceção da fasciculação e flacidez musculares, e preveniu a ocorrência de diarréia (grupo 2). O tratamento com pralidoxima (grupo 3) mostrou-se altamente eficaz, impedindo a manifestação de todos os sinais.

\section{Determinação da Atividade da Colinesterase Plasmática}

Os animais tratados com paraoxon (grupo 1) e paraoxon mais atropina (grupo 2) apresentaram resultados de atividade da colinesterase de 32,2\% e $43,8 \%$, respectivamente, revelando diferenças estatisticamente significativas $(\mathrm{p}<0,01)$ em relação ao grupo-controle. Já os animais tratados com paraoxon mais pralidoxima (grupo 3) apresentaram atividade da colinesterase de $94,1 \%$, não havendo diferença estatisticamente significativa em relação ao grupocontrole (Tabela 2).

\section{Histopatologia}

A análise histopatológica mostrou nos animais dos grupos tratados com paraoxon (grupo 1) e paraoxon mais atropina (grupo 2) a presença de fibras musculares necróticas, freqüentemente com fagocitose, que se encontravam próximas umas às outras nas áreas mais afetadas. Essas fibras foram evidenciadas com maior nitidez na reação histoquímica para fosfatase ácida (Fig. 1). A reação de NADH-TR mostrou fibras com diferentes graus de alteração da trama intermiofibrilar, correspondendo a alterações da estrutura normal das fibras muscula-

Tabela 1 - Sinais observados durante o experimento, em ordem de aparecimento, sendo (+) leve, $(++)$ moderado e $(+++)$ intenso.

\begin{tabular}{lcccc}
\hline Sinal & $\begin{array}{c}\text { Grupo } 1 \\
\text { Paraoxon }\end{array}$ & $\begin{array}{c}\text { Grupo 2 } \\
\text { Paraoxon }+ \\
\text { atropina }\end{array}$ & $\begin{array}{c}\text { Grupo 3 } \\
\text { Paraoxon }+ \\
\text { pralidoxima }\end{array}$ & $\begin{array}{c}\text { Grupo } 4 \\
\text { Controle }\end{array}$ \\
\hline Salivação aumentada & +++ & + & - & - \\
Lacrimejamento & +++ & + & - & - \\
Exoftalmia & +++ & ++ & - & - \\
Tremores & +++ & ++ & - & - \\
Dispnéia & +++ & + & - & - \\
Fasciculação muscular & +++ & +++ & - & - \\
Ataxia & +++ & ++ & - & - \\
Prostração & +++ & + & - & - \\
Diarréia & +++ & - & - & - \\
\hline
\end{tabular}


Tabela 2 - Atividade da colinesterase plasmática $(\Delta \mathrm{E} / \mathrm{ml} / \mathrm{min})$ e desvios-padrão.

\begin{tabular}{lcccc}
\hline & $\begin{array}{c}\text { Grupo 1 } \\
\text { Paraoxon }\end{array}$ & $\begin{array}{c}\text { Grupo 2 } \\
\text { Paraoxon } \\
\text { + atropina }\end{array}$ & $\begin{array}{c}\text { Grupo 3 } \\
\text { Paraoxon } \\
\text { + pralidoxima }\end{array}$ & $\begin{array}{c}\text { Grupo 4 } \\
\text { Controle }\end{array}$ \\
\hline $\begin{array}{l}\text { Atividade } \\
\%\end{array}$ & $0,48 \pm 0,092$ & $0,65 \pm 0,124$ & $1,38 \pm 0,241$ & $1,46 \pm 0,133$ \\
& 32,2 & 43,8 & 94,1 & 100,0 \\
\hline
\end{tabular}

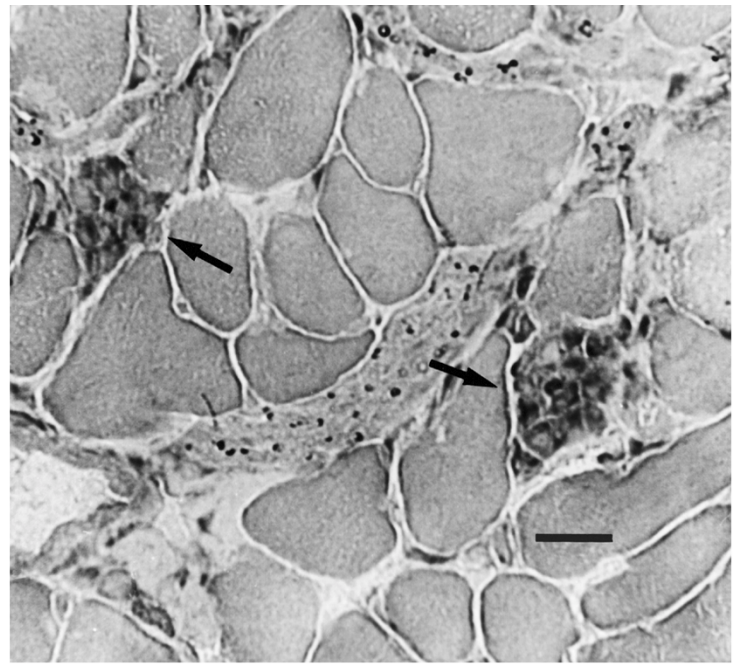

Figura 1 - Corte transversal de fibras musculares exibindo necrose e fagocitose de algumas delas (setas). Músculo diafragma de rato intoxicado pelo paraoxon. Reação histoquímica pela fosfatase ácida. A barra corresponde a 100 $\mu \mathrm{m}$.

res (Fig. 2). Com menor freqüência foram observadas fibras atróficas angulares. No grupo tratado com paraoxon mais pralidoxima (grupo 3), fibras necróticas e com alteração da trama intermiofibrilar foram encontradas em pequeno número. Os resultados da análise histopatológica dos músculos diafragma dos animais estudados (analisados em conjunto em cada grupo), expressos semiquantitativamente, encontram-se na Tabela 3.

\section{DISCUSSÃO}

Embora o comprometimento do sistema muscular esquelético em intoxicações por alguns organo-

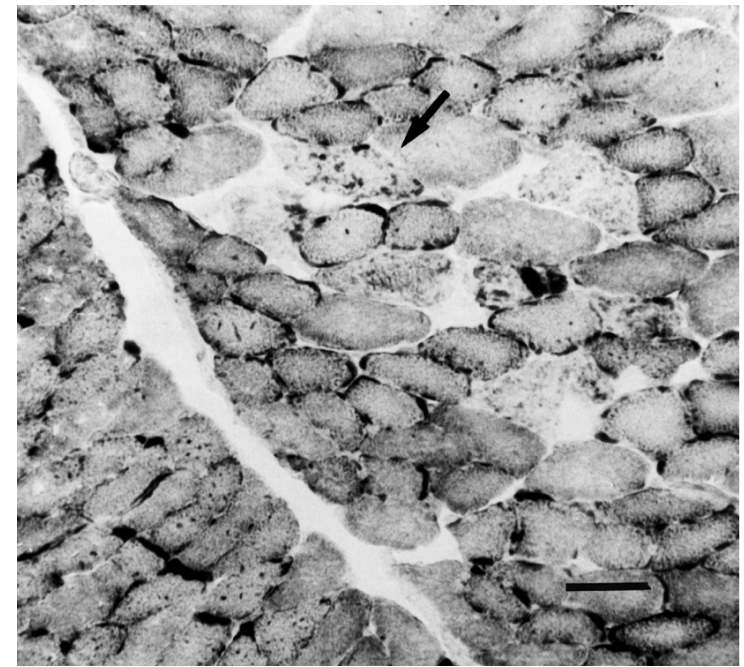

Figura 2 - Corte transversal de fibras musculares exibindo alteração da estrutura interna das mesmas (a seta indica uma destas fibras). Músculo diafragma de rato intoxicado pelo paraoxon. Reação histoquímica pela Nicotinamida-adeninadinucleotídeo-tetrazolium-redutase (NADH-TR). A barra corresponde a $50 \mu \mathrm{m}$.

fosforados seja conhecido de longa data, pouca importância se dá a este fato, haja vista que a avaliação rotineira por estes compostos não inclui protocolos de miotoxicidade. Sabe-se que a principal causa de morbidade e mortalidade nas intoxicações pelos organofosforados é o comprometimento cardiorrespiratório. No entanto, é provável que o comprometimento da musculatura esquelética, sobretudo da musculatura respiratória, contribua tal morbidade, o que reforça a importância da avaliação das lesões musculares nessas intoxicações.

Experimentalmente, além dos sinais de intoxicação típicos dos organofosforados, o parâmetro mais utilizado na avaliação do nível de toxicidade desses

Tabela 3 - Alterações encontradas no músculo diafragma em áreas selecionadas, expressas de forma semiquantitativa: (+) até $5 \%$ de fibras alteradas; $(++)$ de 5 a $15 \%$ de fibras alteradas; $(+++)$ acima de $15 \%$ de fibras alteradas.

\begin{tabular}{lcccc}
\hline Alterações & $\begin{array}{c}\text { Grupo 1 } \\
\text { Paraoxon }\end{array}$ & $\begin{array}{c}\text { Grupo 2 } \\
\text { Paraoxon } \\
+ \text { atropina }\end{array}$ & $\begin{array}{c}\text { Grupo 3 } \\
\text { Paraoxon } \\
\text { + pralidoxima }\end{array}$ & $\begin{array}{c}\text { Grupo 4 } \\
\text { Controle }\end{array}$ \\
\hline Necrose/fagocitose & +++ & +++ & + & - \\
Desorganização da trama intermiofibrilar & +++ & +++ & + & - \\
Fibras atróficas angulares & + & + & - & - \\
\hline
\end{tabular}


compostos é a medida da atividade da colinesterase plasmática. As doses utilizadas em nosso experimento foram suficientes para desencadear sinais de intoxicação, bem como diminuição da atividade da colinesterase plasmática, o que permitiu avaliar claramente as diferenças entre os grupos tratados.

Com relação aos antídotos, a atropina age como inibidor competitivo da acetilcolina em nível dos receptores muscarínicos, porém não tem ação nos receptores nicotínicos, que são os existentes na placa motora. Isto explica o fato de que a atropina não impediu o desenvolvimento da miopatia no diafragma. Dekleva e col. ${ }^{8}, 1989$, descreveram resultados semelhantes em experimentos com ratos intoxicados pelo organofosforado soman, nos quais verificaram que a atropina não prevenia o desenvolvimento da miopatia mesmo com doses altas $(20 \mathrm{mg} / \mathrm{kg})$.

No presente estudo, o uso da pralidoxima imediatamente após a administração do paraoxon impediu a inibição da colinesterase plasmática e preveniu o desenvolvimento da miopatia. Ariens e col. ${ }^{2}, 1969$, em trabalho pioneiro sobre miopatia por organofosforados em ratos, já haviam relatado o papel protetor da pralidoxima com relação a este efeito sobre o músculo esquelético. Em humanos, tem sido relatado que as oximas são capazes de reativar a colinesterase por um período que varia de 24 a 36 horas após a intoxicação ${ }^{11}$. No entanto, não há relatos quanto ao seu papel preventivo da miopatia no homem.

No que se refere à padronização das técnicas histoquímicas para avaliação das lesões muscula-

\section{REFERÊNCIAS BIBLIOGRÁFICAS}

1. AHLGREN, J.D.; MANZ, H. J.; HARVEY, J. C. Myopathy of chronic organophosphate poisoning: a clinical entity? South Med, J., 72: 555-63, 1979.

2. ARIENS, A. T.; MEETER, E.; WOLHIUS, O. L.; VAN BENTHEN, R. M. J. Reversible necrosis at the end-plate region in striated muscles of the rat poisoned with cholinesterase inhibitors. Experientia, 25: 57-9, 1969.

3. BRIGTH, J. E.; INNS, R. H.; TUCKWELL, N. J.; GRIFFTHS, G. D.; MARRS, T. C. A histochemical study of changes observed in the mouse diaphragm after organophosphate poisoning. Hum. Exp. Toxicol., 10: 9-14, 1991.

4. DE BLEECKER, J. L.; WILLEMS, J.; DE REUCK, J., SANTENS. P.; LISON, D. Histological and histochemical study of paraoxon myopathy in the rat. Acta Neurol. Belg., 91: $255-70,1991$

5. DE BLEECKER, J. L.; VAN DEN ABEELE, K. G.; DE REUCK, J. L. Variable muscle involvement of rat skeletal muscles in paraoxon-induced necrotizing myopathy. Res. Chem. Pathol. Com. Pharmacol., 75: 309-22, 1992. res, os presentes resultados indicaram que a utilização da reação histoquímica para fosfatase ácida mostra, com bastante nitidez, as fibras alteradas nesta miopatia, já que evidencia atividade lisossomal e, portanto, processo necrótico. A reação para a enzima oxidativa NADH-TR também mostrou-se útil por demonstrar claramente fibras com alteração da trama intermiofibrilar. Os vários autores citados na literatura, que estudaram experimentalmente a miopatia por organofosforados, utilizaram outras técnicas histológicas e histoquímicas, especialmente a coloração pelo tricrômico modificado de Gomori $^{4,13,14,15,17}$. Brigth e col. ${ }^{3}, 1991$, estudaram a expressão da fosfatase ácida em músculos de animais intoxicados, porém sem finalidade de quantificar as lesões.

Em conclusão, a proposta foi avaliar o grau de miotoxicidade de organofosforados através de testes em ratos albinos, utilizando doses subletais capazes de produzir sinais de intoxicação e diminuição da atividade da colinesterase, com ou sem uso de antídotos. A análise do músculo diafragma, através de congelação ultra-rápida e avaliação semiquantitativa das lesões musculares em cortes transversais seriados, corados por uma bateria mínima e suficiente de técnicas histológicas e histoquímicas (hematoxilinaeosina, fosfatase ácida e NADH-TR), mostrou-se satisfatória. Baseados nestas observações, considerou-se esta metodologia adequada para avaliação de miotoxicidade por diferentes produtos organofosforados, que pode ser realizada rotineiramente em testes toxicológicos.

6. DE REUCK, J. \& WILLEMS, J. Acute parathion poisoning: myopathic changes in the diaphragm. J. Neurol., 208: 30914, 1975.

7. DE REUCK, J.; COLARDYN, F.; WILLEMS, J. Fatal encephalopathy in acute poisoning with organophosphorus insecticides: a clinico-pathologic study of two cases. 1979. Clin. Neurol. Neurosurg., 81: 247-54, 1979.

8. DEKLEVA, A.; SKET, D.; SKETLJ, J.; BRZIN, M. Attenuation of soman-induced lesions of skeletal muscle by acethylcholinesterase reactivating and non-reactivating antidotes. 1989. Acta Neuropathol., 79: 183-9, 1989.

9. DETTBARN, W-D. Pesticide induced muscle necrosis: mechanisms and prevention. Fund. Appl. Toxicol., 4: S18S26, 1984

10. DUBOWITZ, V. Muscle biopsy: a practical approach. 2nd ed. London, Bailliere Tindall, 1985.

11. ECOBICHON; D. J. \& JOY, R. M. Pesticides and neurological diseases. In: Casarett, L. J. \& Doull, J. Toxicology the basic science of poisons. 4th ed. Boca Raton, CRC Press, 1991. p. 565-622. 
12. ELLMAN, G. L.; COUTNEY, K. D.; ANDRES Jr, V.; FEATHERSTONE, R. M. A new rapid colorimetric determination of acethylcholinesterase activity. Biochem Pharmacol., 7: 88-95, 1961.

13. FENICHEL, G. M.; KIBLER, W. B.; WILLIAM, H.; OLSON, M. D.; DETTBARN, W-D. Chronic inhibition of cholinesterase as a cause of myopathy. Neurology, 22: 102633, 1972.

14. GUPTA, R. C.; PATTERSON, G. T.; DETTBARN, W-D. Mechanisms of toxicity and tolerance to diisopropylfluoridate at the neuromuscular junction of the rat. Toxicol. Appl. Pharmacol., 84: 541-50, 1986.

15. LASKOWSKI, M. D.; OLSON, M. H.; DETTBARN, W-D. Ultrastructural changes of the motor end-plate produced by an irreversible cholinesterase inhibitor. Exp. Neurol., 47: 290-306, 1975.
16. UNICAMP/ANDEF/SINDAG. Perfil epidemiológico das intoxicações ocorridas nos Centros de Toxicologia de Belo Horizonte, Campinas, Florianópolis, Ribeirão Preto, Londrina e Maringá, relatório do projeto CCI, Campinas, 1994.

17. WECKER, L. \& DETTBARN, W-D. Paraoxon induced myopathy: muscle specificity and acethylcholine involvement. Exp. Neurol., 51: 281-91, 1976.

18. WECKER, L., MRAK, R. E.; DETTBARN, W-D. Evidence of necrosis in human intercostal muscle following inhalation of an organophosphate insecticide. J. Environ. Pathol. Toxicol. Oncol., 6: 171-5, 1985.

19. WILHEIM, K. Determination of human plasma cholinesterase activity by adapted Ellman method. Arh. Hig. Rad. Tokskol., 19: 199-207, 1967. 